\title{
¿Qué darle a un paciente con sospecha de contagio por COVID-19?
}

\author{
Roberto Guillermo Calva y Rodríguez ${ }^{\ddagger * *}$ \\ ‡ Presidente de la Academia Mexicana de Pediatría. Ciudad de México, México.
}

Con frecuencia nos preguntan "¿qué me puedo tomar, ya que estuve en contacto con un paciente con COVID?". La respuesta no es tan sencilla, ya que bastaría con decirle que si ha tomado las debidas medidas de precaución como es el aislamiento social, la sana distancia entre personas, el lavado de manos con agua y jabón y el empleo de alcohol hidro-alcohólico, éstas son suficientes para no contraer el virus. Sin embargo, como profesionales de la salud no basta con decirle "coma bien, con abundantes frutas y verduras" y "si se siente mal, tome acetaminofén".

Para la enfermedad por COVID-19, tal y como se ha demostrado en múltiples estudios, cuando los individuos no presentan alguna comorbilidad (hipertensión arterial, diabetes, obesidad, etcétera) y tienen una adecuada nutrición, son muy elevadas las posibilidades de tolerar la infección y salir adelante, particularmente en los niños. ${ }^{1-4}$

A pesar de lo anterior, se ha mostrado que, de acuerdo con la última Encuesta Nacional de Salud, nuestra población presenta diversos grados de deficiencias nutricionales, especialmente relacionadas con el hierro, zinc y vitamina $\mathrm{D}$, que los hacen más susceptibles a presentar problemas infecciosos. ${ }^{5}$ En la misma encuesta se señala que en México existen 8.6 millones de personas con diabetes mellitus y 15.2 millones con hipertensión arterial; mientras que el porcentaje de sobrepeso y obesidad a los 20 años es de $37.15 \%$, y en adultos puede llegar a $75.2 \%,{ }^{5}$ los cuales

\footnotetext{
*Correspondencia: RGCR, dr.robertocalvarodriguez@gmail.com Conflicto de intereses: El autor declara que no tiene.

Citar como: Calva y Rodríguez RG. ¿Qué darle a un paciente con sospecha de contagio por COVID-19? Rev Mex Pediatr. 2020; 87(2):79-80 doi: 10.35366/94173

[What to give to a patient suspected of being infected with COVID-19?]
}

son factores asociados con mal pronóstico cuando se adquiere esta infección.

Hasta la fecha no hay medicamentos o suplementos nutricionales que hayan demostrado ser efectivos para el tratamiento o para la prevención de la infección por COVID $-19 .{ }^{6}$ No obstante, se han establecido hipótesis relacionadas con las vitaminas $\mathrm{D}, \mathrm{C}$ y el zinc para determinar si pueden ayudar a mejorar la respuesta inmune en pacientes con COVID-19. Si se comprobara su efectividad, entonces podría ser una alternativa para el manejo de pacientes con sospecha de esta infección.

Se conoce que la vitamina $\mathrm{C}$ mejora la inmunidad al estimular la producción de interferón, también aumenta la proliferación de linfocitos, de neutrófilos, así como la capacidad de fagocitosis. Además, la vitamina $\mathrm{C}$ tiene funciones pleiotrópicas en la modulación del sistema inmune ${ }^{7}$ y propiedades antioxidantes, por lo cual se ha propuesto como adyuvante para pacientes en estado crítico, en quienes los niveles de vitamina $\mathrm{C}$ se reducen. ${ }^{8,9}$ En una revisión sistemática se señala que en ensayos controlados con placebo se ha demostrado que la vitamina $\mathrm{C}$ reduce la duración de los resfriados. ${ }^{10}$ De ahí que, la suplementación para pacientes con COVID-19 podría ser prometedor.

En cuanto a la vitamina D, se sabe que induce la secreción de péptidos antimicrobianos y ha demostrado que modula el sistema inmune innato y adaptativo; mientras que su deficiencia se asocia con disminución de la autoinmunidad y mayor susceptibilidad a infecciones. ${ }^{11,12}$ Se ha destacado el papel de la vitamina D en la reducción del riesgo de enfermedades respiratorias, ya que induce la producción de péptidos antimicrobianos (como catelicidinas y defensinas), los cuales pueden reducir la tasa de replicación viral e impedir el aumento de citocinas proinflamatorias. Esto último podría ser relevante en pacientes con infección grave por COVID-19, 
por lo que en la actualidad se están desarrollando varios ensayos clínicos para determinar si la suplementación de vitamina $\mathrm{D}$ puede resultar benéfica.

Por su parte, el zinc es un oligoelemento necesario para el sistema inmune. Se ha observado que cuando los pacientes tienen deficiencia presentan disfunciones inmunes graves, ${ }^{13}$ así como pérdida de sabor. ${ }^{14,15}$ Además, hay que destacar que el zinc es un inhibidor de varios virus, como los coronavirus. ${ }^{16}$ Entonces, debido a estas acciones del zinc, es posible que pueda ser otra alternativa para la infección por COVID-19, por lo que ya se han comenzado estudios para determinar su posible efectividad. Uno de ellos evalúa la administración de zinc intravenoso y se desarrolla en Australia; en otros ensayos clínicos se combina la administración de zinc con hidroxicloroquina, vitamina $\mathrm{D}$ y vitamina $\mathrm{C}$.

En conclusión, por el momento, la mejor recomendación es mantener las medidas de higiene generales mientras disponemos de los resultados de los ensayos clínicos que se están desarrollando, para determinar si la vitamina $\mathrm{C}$, vitamina $\mathrm{D}$ o el zinc pueden brindar algún beneficio para la infección de COVID-19.

\section{REFERENCIAS}

1. Instituto Mexicano del Seguro Social. Algoritmos interinos para la atención del COVID-19. http://educacionensalud.imss.gob. $\mathrm{mx} / \mathrm{es} / \mathrm{system} / \mathrm{files} /$ Algoritmos_interinos_COVID19_CTEC.pdf. Actualización del 5 de mayo del 2020.

2. Organización Mundial de la Salud. Frequently asked questions: Breastfeeding and COVID-19 for health care workers. 2020. [Acceso 16 de junio de 2020] Disponible en: https://www.who. int/docs/default-source/maternal-health/faqs-breastfeeding-andcovid $-19 . p d f ? s f v r s n=d 839 \mathrm{e} 6 \mathrm{c0} 05$
3. Zhou F, Yu T, Du R et al. Clinical course and risk factors for mortality of adult inpatients with COVID-19 in Wuhan, China: a retrospective cohort study. Lancet. 2020; 395(10229): 1054-1062.

4. Zhang J, Xie B, Hashimoto K. Current status of potential therapeutic candidates for the COVID-19 crisis. Brain Behav Immun. 2020. doi: 10.1016/j.bbi.2020.04.046.

5. Encuesta Nacional de Salud. Instituto nacional de Salud Pública. 2018.

6. Montaño-Luna VE, Pacheco-Rosas DO, Vázquez-Rosales JG, Labra-Zamora MG, Fuentes-Pacheco Y, Sámano-Aviña M et al. Manejo clínico de casos pediátricos de COVID-19. Rev Med IMSS. 2020. Disponible en: http://revistamedica.imss.gob.mx/editorial/ index.php/revista medica/article/view/3702/3736

7. Carr AC, Maggini S. Vitamin C and immune function. Nutrients. 2017; 9(11): 1211.

8. Carr AC, Rosengrave PC, Bayer S, Chambers S, Mehrtens J, Shaw GM. Hypovitaminosis $C$ and vitamin $C$ deficiency in critically ill patients despite recommended enteral and parenteral intakes. Crit Care. 2017; 21(1): 300.

9. May JM, Harrison FE. Role of vitamin $\mathrm{C}$ in the function of the vascular endothelium. Antioxid Redox Signal. 2013; 19(17): 2068-2083.

10. Hemilä $\mathrm{H}$, Chalker $\mathrm{E}$. Vitamin $\mathrm{C}$ for preventing and treating the common cold. Cochrane Database Syst Rev. 2013. CD000980. doi: 10.1002/14651858.CD000980.pib4.

11. Aranow C. Vitamin D and the immune system. J Investig Med. 2011; 59(6): 881-886.

12. Grant WB, Lahore $\mathrm{H}, \mathrm{McD}$ Donnell $\mathrm{SL}$ et al. Evidence that vitamin D supplementation could reduce risk of influenza and COVID-19 infections and deaths. Nutrients. 2020; 12 (4): 988.

13. Prasad AS. Zinc in human health: effect of zinc on immune cells. Mol Med. 2008; 14(5-6): 353-357.

14. Keyhan SO, Fallahi HR, Cheshmi B. Dysosmia and dysgeusia due to the 2019 Novel Coronavirus; a hypothesis that needs further investigation. Maxillofac Plast Reconstr Surg. 2020; 42(1): 9.

15. Doty RL. Treatments for smell and taste disorders: A critical review. Handb Clin Neurol. 2019; 164: 455-479.

16. te Velthuis AJ, van den Worm SH, Sims AC, Baric RS, Snijder EJ, van Hemert MJ. Zn(2+) inhibits coronavirus and arterivirus RNA polymerase activity in vitro and zinc ionophores block the replication of these viruses in cell culture. PLoS Pathog. 2010; 6(11): e1001176. 